\title{
Arthroscopic Diagnosis of Occult Posterolateral Meniscocapsular Separations: Another Hidden Lesion
}

\author{
Robert L. Parisien, M.D., Max Shin, B.A., Lauren M. Boden, M.D., \\ Stephanie Y. Jo, M.D., Ph.D., Lisa Victorius, P.A., Brian J. Sennett, M.D., and \\ Miltiadis H. Zgonis, M.D.
}

\begin{abstract}
Purpose: The purpose of this study was to describe the surgical findings and clinical outcomes in a series of patients with occult posterolateral meniscocapsular separations diagnosed arthroscopically after a negative magnetic resonance imaging (MRI) scan. Methods: A retrospective analysis of prospectively collected data of consecutive patients who underwent surgical arthroscopy with repair of an occult posterolateral meniscocapsular separation by 2 fellowship-trained orthopaedic sports medicine surgeons at a single institution was performed. All lesions were identified arthroscopically in the posterolateral aspect of the lateral compartment as a distinct pathologic separation between the posterolateral capsule and adjacent meniscal tissue with increased excursion on probing. Clinical examination notes, MRI scans, and operative reports were reviewed. Patient-reported outcome measures were assessed via patient questionnaire. Results: A total of 6 patients were included for analysis. MRI evaluation of the lateral meniscus was unrevealing in 4 patients, suggesting a possible tear of the body of the lateral meniscus in one patient and demonstrating a parameniscal cyst abutting the anterior root of the lateral meniscus in another patient. Arthroscopic examination revealed meniscocapsular separations of the posterolateral meniscus in all 6 knees, with 2 knees demonstrating concomitant bucket-handle meniscus tears. Patientreported outcomes were determined for $67 \%$ of study patients. The average reported International Knee Documentation Committee score was 63.8, the average Knee Outcome Survey Activities of Daily Living Scale score was reported as 63, the 12-Item Short Form Survey (SF-12) Physical score averaged 46.8 with an average SF-12 Mental score of 59.9. Conclusions: The diagnosis of occult posterolateral meniscocapsular separations (MCS) could be missed on advanced imaging, such as MRI, so arthroscopic diagnosis may be required. This study indicates that arthroscopic diagnosis and repair of occult posterolateral MCS results in good functional and clinical outcomes. Level of Evidence: IV, therapeutic case series.
\end{abstract}

$\mathbf{M}$ eniscal tears are the most commonly treated injury of the knee joint and one of the most frequently treated injuries in orthopaedic surgery, with

From the Harvard Medical School (R.L.P.), Boston, Massachusetts, the Perelman School of Medicine at the University of Pennsylvania (M.S.) and the University of Pennsylvania (L.M.B., S.Y.J., L.V., B.J.S., M.H.Z.), Philadelphia, Pennsylvania, U.S.A.

The authors report the following potential conflict of interest or source of funding: R.L.P. is on the editorial board for Arthroscopy, and Arthroscopy, Sports Medicine, and Rehabilitation and is a member of the research committee for the American Orthopaedic Society for Sports Medicine. Full ICMJE author disclosure forms are available for this article online, as supplementary material.

Received August 17, 2020; accepted January 21, 2021.

Address correspondence to Robert L. Parisien, M.D., Harvard Medical School, Boston, MA 02115, U.S.A. E-mail:Robert.L.Parisien@gmail.com

(C) 2021 THE AUTHORS. Published by Elsevier Inc. on behalf of the Arthroscopy Association of North America. This is an open access article under the CC BY-NC-ND license (http://creativecommons.org/licenses/by-nc-nd/4.0/). 2666-061X/201407

https://doi.org/10.1016/j.asmr.2021.01.011 an incidence of $61 / 100,000$ in the United States. ${ }^{1}$ Left untreated, meniscus tears may compromise joint integrity and have been associated with degenerative changes leading to long-term dysfunction. ${ }^{2,3}$ Because of its rigid adherence to the medial collateral ligament and relative lack of excursion, the medial meniscus is more commonly injured as compared to the lateral meniscus, with the posterior horn most often affected. Classically described tear patterns include radial, horizontal, vertical, complex, flap-type, and bucket-handle. ${ }^{4}$

Of the various meniscus tear types, meniscocapsular separations (MCS) are uncommon injuries characterized by detachment of the meniscus from its capsular attachment. This detachment may lead to further instability of the knee with progression of meniscal tear pattern and cartilage injury. ${ }^{5}$ Strobel $^{6}$ first introduced the term "ramp lesion" in 1988 to describe tears involving the meniscocapsular junction at the posterior meniscocapsular zone. Since then, there has been renewed interest in these injuries and the challenges of 
identifying them on magnetic resonance imaging (MRI) and arthroscopy because of their position in the anatomical "blind spot" of the knee. ${ }^{7}$ For instance, the positive predictive values of identifying MCS with MRI has been shown to be as low as $9 \%$ for the medial meniscus and $13 \%$ for the lateral meniscus. ${ }^{8}$ Most of these studies have predominantly focused on MCS of the medial meniscus and often involve concomitant injuries. However, there is only 1 case report in the literature describing an isolated MCS involving the lateral meniscus. ${ }^{9}$ A single case report of a lateral MCS has been described in the setting of a rare anatomic aberration known as a double-layered lateral meniscus. ${ }^{10}$ The authors describe improvement of clinical symptoms after resection of the upper accessory meniscus and repair of the MCS. Additionally, hypermobility of the posterior horn of the lateral meniscus has been recently identified in case reports and limited case series. ${ }^{1-14}$ Although the pathophysiology and optimal treatment have yet to be elucidated, this entity differs from a MCS because it exists in the absence of a discrete tear or traumatic capsular separation and is believed to be due to the disruption of the popliteomeniscal fascicles.

The purpose of this study was to describe the surgical findings and clinical outcomes in a series of patients with occult posterolateral meniscocapsular separations diagnosed arthroscopically after a negative MRI scan. We hypothesize significantly improved functional and clinical outcomes after arthroscopic repair of occult posterolateral MCS.

\section{Methods}

A retrospective analysis of prospectively collected data of consecutive patients who underwent surgical arthroscopy with repair of an occult posterolateral meniscocapsular separation by 2 fellowship-trained orthopaedic sports medicine surgeons at a single institution was performed between March 2016 through March 2020. All patients had an available preoperative MRI scan using a 1.5-T MRI scanner. All MRI sequences were reviewed by one of the senior orthopaedic surgeons and a fellowship-trained musculoskeletal radiologist. All patients underwent a period of nonoperative management consisting of physical therapy and administration of nonsteroidal anti-inflammatory medication. Indications for surgical intervention included objective complaints of mechanical symptoms and persistent lateral joint line (LJL) pain despite nonsurgical management for more than 3 months duration. Exclusion criteria were as follows: ligamentous instability as determined by positive Lachman, positive pivot-shift, positive anterior-posterior drawer, and increased varus-valgus laxity, as well as those patients undergoing surgical arthroscopy for ligamentous reconstruction and cartilage injury. Demographic information including age, sex, and body mass index were collected. Clinical examination notes were reviewed with data collected for chief complaint, laterality, mechanism of injury, time from injury to surgery and pertinent physical exam findings. MRI and operative reports were reviewed with data collected and recorded with regards to meniscus pathology, ligamentous integrity and degree and location of associated articular cartilage damage. All lesions were identified arthroscopically in the posterolateral aspect of the lateral compartment as a distinct pathologic separation between the posterolateral capsule and adjacent meniscal tissue with increased excursion upon probing (Fig 1).

Patient reported outcome measures were assessed via patient questionnaire at greater than one-month postoperatively with evaluation of the International Knee Documentation Committee score (IKDC), Knee Outcome Survey Activities of Daily Living Scale (KOSADL), 12-Item Short Form Survey (SF-12) with inclusion of both the Mental Component Score and Physical Component Score. Descriptive statistics were determined in an attempt to summarize the sample data.

\section{Results}

Six patients were included for analysis. Sex characteristics of the cohort are 5 female and 1 male (Table 1). The average age of the entire cohort was 44.2 years old, with female age range from 31 to 63 years of age and the 1 male patient aged 36 years old. The average body mass index for the cohort was 29.5 (range, 19.1-39.5). There was an equal distribution of knee laterality $(3$ right and 3 left). All patients presented with primary complaints of intermittent mechanical locking of the knee resulting in the inability to achieve full extension with significant concomitant pain. Mechanism of injury was reported as atraumatic in $33.3 \%$ (2 of 6) of patients with the remaining patients reporting injury as the result of squatting, bus accident, exercise and getting out of bed. All patients demonstrated LJL tenderness on physical examination with an average arc of motion of $136.7^{\circ}$ (range, $-5^{\circ}$ to $135^{\circ}$ ). All other physical

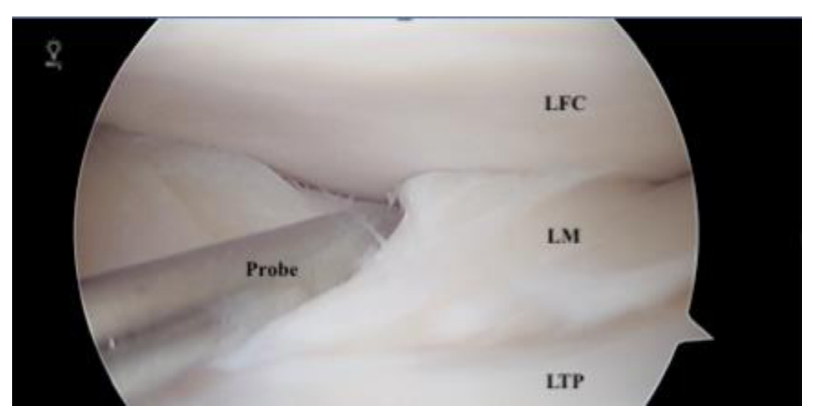

Fig 1. Pathologic lateral meniscocapsular separation with increased meniscal excursion. 
Table 1. Patient Characteristics

\begin{tabular}{llllllllll}
\hline Case & Age & Sex & BMI & Laterality & \multicolumn{1}{c}{ MOI } & Subjective & Joint Line & ROM & Symptoms to Surgery \\
\hline 1 & 31 & F & 24.2 & R & Atraumatic & Locking pain & LJLT & 0 to 120 & 8 months \\
2 & 55 & F & 19.1 & L & Atraumatic & Locking pain & LJLT & -5 to 135 & 4 years \\
3 & 39 & F & 37.1 & L & Squatting & Locking pain & LJLT & -5 to 135 & 12 years \\
4 & 36 & M & 23.6 & L & Bus Accident & Locking pain & LJLT & -5 to 135 & 10 months \\
5 & 41 & F & 33.5 & R & Exercise & Locking pain & LJLT & -5 to 135 & 5 years \\
6 & 63 & F & 39.5 & R & Getting out of bed & Locking pain & LJLT & -5 to 135 & 3 months \\
\hline
\end{tabular}

BMI, body mass index; LJLT, lateral joint line tenderness; MOI, mechanism of injury; ROM, range of motion

examination components, including ligamentous integrity, were unremarkable. The average time from onset of symptoms to arthroscopic evaluation was 45.5 months (range, 3 to 144 months). MRI examination of the lateral meniscus was unrevealing in 4 patients, suggested a possible tear of the body of the lateral meniscus in 1 patient, and demonstrated a parameniscal cyst abutting the anterior root of the lateral meniscus in another patient (Table 2). No evidence of MCS was noted on MRI (Fig 2). However, MRI did reveal lateral compartment articular cartilage pathology in $50 \%$ of affected knees. Arthroscopic examination revealed MCS of the posterolateral meniscus in all 6 knees, with 2 knees demonstrating concomitant bucket-handle meniscus tears (Table 3). All MCS lesions and both bucket handle tears were addressed via arthroscopic repair. Concomitant arthroscopic findings included International Cartilage Regeneration and Joint Preservation Society Grade I pathology of the lateral femoral condyle (LFC) in 3 knees, International Cartilage Regeneration and Joint Preservation Society Grade II in 1 knee, and 2 knees without articular pathology of the LFC. Similarly, diffuse Grade I articular lesions were arthroscopically identified on the lateral tibial plateau in 2 knees. One knee demonstrated a focal Grade II articular defect, 1 knee revealed diffuse Grade III articular changes, and the remaining 2 knees were without articular abnormality.

Patient reported outcomes were determined for $67 \%$ (4 of 6) of study patients (Table 4). The average reported IKDC score was 63.8 (range, 50 to 81 ), with 87 representing the maximum possible score. The average KOS-ADL score was reported as 63 (range, 55 to 68).
The SF-12 Physical score averaged 46.8 (range, 42.7 to 54.2) with an average SF-12 Mental score of 59.9 (range, 58.8 to 60.3 ).

\section{Discussion}

Six consecutive patients underwent arthroscopic repair of an occult posterolateral MCS at an average time of 45.5 months from the initial onset of symptoms. Preoperative MRI revealed varying degrees of articular cartilage damage to the LFC and tibial plateau without evidence of posterolateral MCS. After repair, all patients reported good clinical outcomes with satisfactory IKDC, KOS-ADL, SF-12 Physical, and SF-12 Mental scores. Many studies have designated medial MCS as an occult injury, citing anatomic location and limitations with MRI as the cause for missed diagnosis. ${ }^{7,8,15,16}$ Greif et al. ${ }^{17}$ reviewed anatomic variants of medial MCS with associated MRI findings in an attempt to simplify and standardize the diagnosis. However, the modified classification relies on mechanism of injury, associated ACL injury and associated MRI findings for diagnosis. To our knowledge there is only a single case report involving a patient with an isolated lateral MCS, which was diagnosed via ultrasound scanning after a negative MRI result. ${ }^{\text {? }}$

Much of the literature regarding medial MCS focuses on younger athletes sustaining a twisting injury; however, our patients were middle aged with varied mechanisms of injury. ${ }^{16,18}$ All 6 of our patients presented with lateral knee pain and locking symptoms that persisted despite months to years of conservative therapy. Additionally, our patients had normal examination results aside from LJL tenderness. Persistent

Table 2. MRI Findings

\begin{tabular}{|c|c|c|c|}
\hline Case & Meniscus & Collateral Ligament & Cartilage \\
\hline$\overline{1}$ & Normal & Normal & Normal \\
\hline 2 & Normal & Normal & Chondrosis, LTP \\
\hline 3 & Cyst abutting anterior root, LM & Normal & Normal \\
\hline 4 & Possible small tear, LM body & Normal & Normal \\
\hline 5 & Normal & Normal & Full thickness cartilage defect, LFC \\
\hline 6 & Normal & Normal & Subchondral cyst formation \\
\hline
\end{tabular}

LFC, lateral femoral condyle; LM, lateral meniscus; LTP, lateral tibial plateau. 

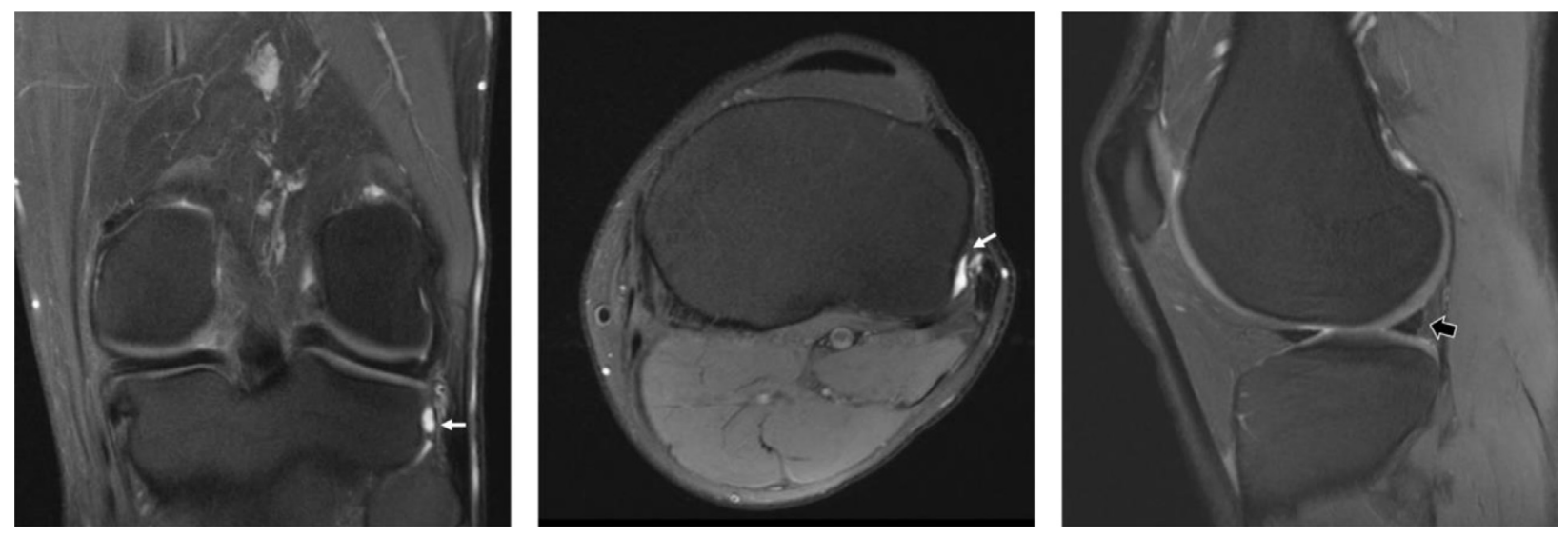

Fig 2. Coronal, axial, and sagittal magnetic resonance imaging sequences without contrast.

joint line tenderness is consistent with previous reports of medial MCS and should raise clinical concern despite the presence of unrevealing imaging. ${ }^{18-19}$ Although subtle findings of cartilage defects and small cysts were identified on MRI in this series, no definitive lateral meniscus tears or MCS were identified on preoperative imaging. One patient's report noted a possible small tear of the body of the lateral meniscus, but there was no evidence of MCS or posterior horn involvement. Despite a fellowship trained musculoskeletal radiologist scrutinizing the images, $100 \%$ of the MCS were missed on imaging in this study. Although a $1.5 \mathrm{~T}$ scanner was used for image analysis, the literature has demonstrated no benefit of $3 \mathrm{~T}$ as compared to $1.5 \mathrm{~T}$ scanners in the ability to diagnose MCS. ${ }^{18,19}$

Arthroscopy provided the definitive diagnosis of lateral MCS for all 6 patients in this study and should be considered the gold standard for diagnosis of this occult lesion. The lengthy average time of 45.5 months (range, 3-144 months) from symptom onset to arthroscopic intervention was secondary to the absence of positive advanced MR imaging resulting in extended conservative nonoperative management. Additionally, degenerative changes to the cartilage of both the LFC and the lateral tibial plateau were found in 4 of $6(66 \%)$ patients. Two of those patients also had associated bucket-

Table 3. Arthroscopic Findings of Lateral Compartment

\begin{tabular}{llll}
\hline Case & \multicolumn{1}{c}{ Meniscus } & ICRS Grade, LFC & ICRS Grade, LTP \\
\hline 1 & MCS & Normal & Normal \\
2 & MCS & Grade 1 & Grade 3, Diffuse \\
3 & MCS and bucket & Grade 1 & Grade 2, Diffuse \\
& handle tear & & Grade 2, Diffuse \\
4 & MCS and bucket & Grade 2 & \\
& handle tear & Grade 1 & Grade 2, Focal \\
5 & MCS & Normal & Normal \\
\hline
\end{tabular}

ICRS, International Cartilage Regeneration and Joint Preservation Society; LFC, lateral femoral condyle; LTP, lateral tibial plateau; MCS, meniscocapsular separation. handle tears suggesting that a classification system similar to that for medial MCS may be warranted. ${ }^{20}$ Understanding the intricate anatomy of the posterior horn of the lateral meniscus further explains why this pathology is persistently symptomatic and difficult to diagnose on imaging. Cadaveric analysis has demonstrated attachment of the posterolateral capsule to the superior $11 \%$ of the posterior horn of the lateral meniscus with a length of $16.7 \mathrm{~mm}$, which is a smaller area than the attachment of the medial meniscocapsular junction. ${ }^{15}$

A secondary goal of this study was to report on initial outcomes after arthroscopic repair of occult lateral MCS. Although postoperative subjective outcomes data were unavailable for two of the six patients, progress notes from their last office visits reported that one patient was improving well and the other had no residual symptoms or limitations, indicating positive response to surgical repair of the lateral MCS. Outcomes for the remaining four patients were recorded after surgery at the time of the study. IKDC scores averaged 63.8 (range, 50-81), which is slightly less than that reported by younger patients after repair of ramp lesions but is consistent with age- and gender-based normative data. ${ }^{18,21,22}$ The SF-12 physical scores in this series were

Table 4. Patient-Reported Outcomes

\begin{tabular}{lccccc}
\hline & & & SF-12 & SF-12 \\
Case & Follow-up (mo) & IKDC & KOS-ADL & Physical & Mental \\
\hline $1^{*}$ & 3 & - & - & - & - \\
2 & 7 & $71 / 87$ & $67 / 70$ & 54.2 & 58.8 \\
3 & 39 & $81 / 87$ & $68 / 70$ & 44.2 & 60.5 \\
$4^{*}$ & 26 & - & - & - & - \\
5 & 1 & $50 / 87$ & $55 / 70$ & 45.9 & 60.1 \\
6 & 18 & $53 / 87$ & $62 / 70$ & 42.7 & 60.3 \\
\hline \multicolumn{7}{l}{ SF-12 United States Average $=50}$. \\
IKDC, International Knee Documentation Score; KOS-ADL, Knee \\
Outcome Survey-Activities of Daily Living; SF-12, 12-Item Short \\
Form Survey. \\
*Patients unable to be contacted to administer questionnaires.
\end{tabular}


just below average; however, the mental scores were above average based on the population average of 50 by inherent design of the scoring system. ${ }^{23}$ Additionally, the KOS-ADL scores were excellent with an average of 60.8, indicating minimal disruption in ADLs. These subjective outcomes indicate that patients with occult posterolateral MCS do very well after surgical repair, with overall above average quality of life when compared to norms.

\section{Limitations}

This study is not without limitations. As a small retrospective case series, there is inherent selection bias; however, this was minimized by the consecutive nature of patient inclusion. Comparing the results of surgery at different time points introduces bias. Also, 1 month of follow-up may be too short a time period to fully evaluate the clinical results of surgery. The prospective collection of PRO data would have strengthened the study findings and allowed us to quantitatively analyze the degree of subjective improvement in patient outcomes. This would have also allowed for increased homogeneity in reporting, thus aiding the interpretation of these findings. However, we did not attempt to collect PRO data retrospectively at all time points as this would have contributed to significant recall bias in patients further removed from their index arthroscopic procedure.

\section{Conclusion}

The diagnosis of occult posterolateral MCS could be missed on advanced imaging, such as MRI, so arthroscopic diagnosis may be required. This study indicates that arthroscopic diagnosis and repair of occult posterolateral MCS results in good functional and clinical outcomes.

\section{References}

1. Raj MA, Bubnis MA. Knee Meniscal Tears. StatPearls Publishing. Treasure Island, FL, 2020 Jan.

2. Ihn JC, Kim SJ, Park IH. In vitro study of contact area and pressure distribution in the human knee after partial and total meniscectomy. Int Orthop 1993;17:214-218.

3. Englund M, Roos EM, Roos HP, Lohmander LS. Patientrelevant outcomes fourteen years after meniscectomy: Influence of type of meniscal tear and size of resection. Rheumatology (Oxford) 2001 Jun;40:631-639.

4. Maffulli N, Longo UG, Campi S, Denaro V. Meniscal Tears. Open Access J Sports Med 2010 Apr 26;1:45-54.

5. Joshi A, Usman S, Sabnis B, Kini A. Repairing Posteromedial Meniscocapsular Separation: A Technique Using Inside-Out Meniscal Repair Needles. Arthrosc Tech 2016 Jan 1 1;5:e23e25.

6. Strobel M. Manual of Orthopedic Surgery. New York: Springer, 1988.

7. Sonnery-Cottet B, Conteduca J, Thaunat M, Gunepin FX, Seil R. Hidden lesions of the posterior horn of the medial meniscus: A systematic arthroscopic exploration of the concealed portion of the knee. Am J Sports Med 2014 Apr;42:921-926.

8. Rubin DA, Britton CA, Towers JD, Harner CD. Are MR imaging signs of meniscocapsular separation valid? Radiology 1996 Dec;201:829-836.

9. Schroeder A, Musahl V, Urbanek C, Onishi K. Dynamic Sonographic Visualization of an Occult Posterolateral Meniscocapsular Separation: A Case Report. PM R 2018 Nov; 10:1288-1291.

10. Fukuda A, Nihimura A, Nakazora S, Kato K, Sudo A. Double-layered Lateral Meniscus Accompanied by a Meniscocapsulr Separation. Case Rep Orthop 2015;2015: 357463.

11. Nair R, Dubey N. MR Imaging of the Hypermobile Lateral Meniscus of the Knee: A Case Report. Acta Med Acad 2019 Aug;48:225-229.

12. Steinbacher G, Alentorn-Geli E, Alvarado-Calderon M, Barastegui D, Alvaraz-Diaz P, Cugat R. Knee Surg Sports Traumatol Arthrosc 2019 Feb;27:354-360.

13. Kamiya T, Suzuki T, Otsubo H, Kuroda M, Matsumura T, Kubota C, Yamashita T. Midterm outcomes after arthroscopic surgery for hypermobile lateral meniscus in adults: Restriction of paradoxical motion. J Orthop Sci 2018 Nov;23:1000-1004.

14. Van Steyn MO, Mariscalco MW, Pedroza AD, Smerek J, Kaeding CC, Flanigan DC. The hypermobile lateral meniscus: A retrospective review of presentation, imaging, treatment, and results. Knee Surg Sports Traumatol Arthrosc 2016 May;24:1555-1559.

15. Aman ZS, DePhillipo NN, Storaci HW, Moatshe G, Chahla J, Engebretsen L, LaPrade R. Quantitative and Qualitative Assessment of Posterolateral Meniscus Anatomy. Am J Sports Med 2019 Jul;47:1797-1803.

16. Alessio-Mazzola M, Lovisolo S, Capello AG, Zanirato A, Chiarlone F, Formica M, Felli L. Management of ramp lesions of the knee: a systematic review of the literature. Musculoskeletal Surg 2020 Aug;104:125-133.

17. Greif DN, Baraga MG, Rizzo MG, Mohile NV, Silva FD, Fox T, Jose J. MRI appearance of the different ramp lesion types, with clinical and arthroscopic correlation. Skeletal Radiol 2020 May;49:677-689.

18. Hetsroni I, Lillemoe K, Marx RG. Small Medial Meniscocapsular Separations: A Potential Cause of Chronic Medial-Side Knee Pain. Arthroscopy 2011 Nov;27: 1536-1542.

19. Hirtler L, Unger J, Weninger P. Acute and chronic menisco-capsular separation in the young athlete: diagnosis, treatment and results in thirty seven consecutive patients. Int Orthop 2015 May;39:967-974.

20. Thaunat M, Fayard JM, Guimaraes TM, Jan N, Murphy CG, Sonnery-Cottet B. Classification and Surgical Repair of Ramp Lesions of the Medial Meniscus. Arthrosc Tech 2016 Aug 8;5:e871-e875.

21. Thaunat M, Jan N, Fayard JM, Kajetanek C, Murphy CJ, Pupim B, Gardon R, Sonnery-Cottet B. Repair of Meniscal Ramp Lesions Through a Posteromedial Portal During Anterior Cruciate Ligament Reconstruction: Outcome Study With a Minimum 2-Year Follow-up. Arthroscopy 2016 Nov;32:2269-2277. 
22. Anderson AF, Irrgang JJ, Kocher MS, Mann BJ, Harrast JJ. The International Knee Documentation Committee Subjective Knee Evaluation Form: normative data. Am J Sports Med 2006 Jan;34:128-135.
23. Ware J Jr, Kosinski M, Keller SD. A 12-Item Short-Form Health Survey: construction of scales and preliminary tests of reliability and validity. Med Care 1996 Mar;34: 220-233. 